\title{
A smart classifier for extracting environmental data from digital image time-series: applications for PhenoCam data in a tidal salt marsh
}

Jessica L. O'Connell ${ }^{\mathrm{a}, \mathrm{b}}$ and Merryl Alber ${ }^{\mathrm{a}}$

a Department of Marine Sciences, University of Georgia, Athens, GA 30602-3636; ${ }^{\mathrm{b}}$ Corresponding author email: jessica.oconnell@uga.edu; jessica.oconnell@okstate.edu

\begin{abstract}
PhenoCams are part of a national network of automated digital cameras used to assess vegetation phenology transitions. Effectively analyzing PhenoCam time-series involves eliminating scenes with poor solar illumination or high cover of non-target objects such as water. We created a smart classifier to process images from the "GCESapelo" PhenoCam, which photographs a regularly-flooded salt marsh. The smart classifier, written in R, assigns pixels to target (vegetation) and non-target (water, shadows, fog and clouds) classes, allowing automated identification of optimal scenes for evaluating phenology. When compared to hand-classified validation images, the smart classifier identified scenes with optimal vegetation cover with $96 \%$ accuracy and other object classes with accuracies ranging from $86-100 \%$. Accuracy for estimating object percent cover ranged from 74 to $100 \%$. Pixel-classification with the smart classifier outperformed previous approaches (i.e. indices based on average color content within ROIs) and reduced variance in phenology index time-series. It can be readily adapted for other applications.
\end{abstract}

Keywords: Flooding; Georgia Coastal Ecosystems LTER, PhenoCam; Phenology; Salt Marsh; Tides; Wetlands; Abbreviations: Blue Chromatic Coordinate (BCC); Green Chromatic Coordinate (GCC); Red Chromatic Coordinate (RCC); Red, Green, Blue (RGB) color space; Region of Interest (ROI); Weather Filtering Index (WFI);

\section{Introduction}

Digital images are increasingly used for environmental analyses, including interpreting sediment profile images 
(Romero-Ramirez et al., 2013) and vegetation characteristics (Zhou and Robson, 2001). The National PhenoCam Network (phenocam.sr.unh.edu) uses imagery to record plant phenology across ecosystems and climate zones (Richardson et al., 2007). Image color information is extracted to generate a time-series of phenological indices that track spring vegetation green-up and fall senescence. However, poor solar illumination and non-target objects (flooding, shadows, fog) interfere with vegetation analysis. Here we present an algorithm called the "smart classifier" for classifying imagery pixels, which we developed to automatically process PhenoCam images from a tidal salt marsh.

Extracting ecological data, such as phenology, from digital repeat photography has known challenges. First, noise is introduced when scene illumination changes, altering color information. To reduce illumination noise, one can limit analyses to mid-day, when solar illumination is greatest. However, clouds and rain also change image illumination. To account for this, some explicitly filter scenes with poor illumination. For example, Sonnentag et al. (2012) developed the 90th percentile method, which uses a 3-day moving average window to identify bright, presumably optimal, scenes. This method assumes clouds, fog and rain have a unidirectional influence on imagery digital values, which we will show is not the case. Others created indices meant to flag scenes with poor solar illumination. The Blue Chromatic Coordinate (BCC) indexes large deviations from mean seasonal patterns in blueness within vegetation (Julitta et al., 2014), whereas the Weather Filtering Index (WFI) identifies clouds as differences in the proportion of red and blue in the sky (Ide and Oguma, 2010). However, validation of these weather indices were not in the literature. We wanted to either validate or improve them for our PhenoCam site.

Another challenge for analyzing PhenoCam imagery is eliminating non-target objects such as shadows, snow, fog, or flooding, each of which can cover vegetation (Fig. 1). To our knowledge, only Julitta et al. (2014) classified pixels within PhenoCam scenes, distinguishing between snow and vegetation. Here, we expand this approach to quantify broader environmental variability. Explicitly identifying pixels is especially important for landscapes with high nontarget object cover, such as regularly flooded tidal marshes.

In this paper we describe a smart classifier for PhenoCam images that uses within-scene content to classify pixels as vegetation, water, shadows, fog and clouds. We used the classifier to identify images containing optimal data for phenology analysis (flood-free scenes taken during clear conditions) in order to reduce variability in phenological monitoring. The smart classifier also can produce time-series of flooding, cloud cover and other objects. This approach 
can be extended to other applications.

\section{Materials and Methods}

Digital imagery dataset. We obtained repeat digital images from the "GCESapelo" PhenoCam (StarDot NetCam SC 5MP IR, StarDot Technologies, Buena Park, CA USA), which is mounted on an eddy covariance flux tower operated by the Georgia Coastal Ecosystems Long-term Ecological Research program. The camera collects oblique images of a Spartina alterniflora salt marsh. Tides are semi-diurnal with a range of $\sim 1.2 \mathrm{~m}$ on the marsh platform. Images are collected every half-hour during daylight. We analyzed images from 9/17/13 through 10/15/15 except when the camera was off-line 7/16/14 through 9/2/14. To reduce solar illumination variation, analyses were limited to images collected between 10:00 and 14:30 (6987 scenes).

Analytical approach. To develop a tool that quantifies objects within PhenoCam imagery, we created image masks for both marsh and sky regions of interest (ROIs) (Fig. 1). Next we used the biOps package in R (Bordese and Alini, 2013) to read masks and PhenoCam jpeg images as numerical arrays representing the amount of red, green, and blue (RGB, on a scale of 0-255) in each pixel.

We used the "clustering for large objects" (CLARA) (Kaufman and Rousseeuw, 2009) R function in package "cluster" to build a library of colors for common objects, including the range of digital numbers and color ratios exhibited by each. Sky pixels were classified as clouds or sky, whereas marsh pixels were vegetation, water, shadows, or fog/cloud reflections within water (hereafter "fog" for simplicity). However, we could not tune this unsupervised classification for accuracy because object color spaces overlapped and changed both seasonally and contextually. Therefore, we developed the smart classifier.

To create a smart classifier rule set we initially used the color space of objects from cluster analysis (Fig. 2 Step 1). To assign pixels to objects we found it more efficient to begin by assigning all pixels to the most common object (vegetation), and then subtracting pixels whose color space corresponded to other objects (Fig. 2 Step 2). Next, we compared our results to a subset of 201 randomly selected hand-classified images (training data) (Fig. 2 Step 3). For hand-classification, we visually estimated presence/absence and percent cover quartiles of each object category $(0 \%$ (i.e. absent), $>0-25 \%, 25-50 \%, 50-75 \%, 75-99 \%,>99 \%$ (i.e. full cover)). We used a contingency table approach to assess model output in terms of critical metrics (Kuhn, 2008; Bennett et al., 2013), including accuracy (correctly classified presences and absences/total number of samples * 100), as well as sensitivity (true positives: correctly 
classified presences/total observed presences $* 100)$ and specificity (true negatives: correctly classified absences/ total observed absences * 100). We calculated quantity accuracy based on $\%$ cover, considering an object misclassified if the $\%$ cover from the smart classifier differed by more than $20 \%$ from the mid-point of each quartile. Finally, we calculated sensitivity for vegetation based on scenes hand-classified as $>99 \%$ cover, as these represent optimal imagery for phenology analyses.

We iteratively tuned the classification algorithm until detection accuracy for each object class was $>90 \%$ across training images (Fig. 2 Step 4). Tuning involved reducing misclassified pixels by modifying object color space in different situations. When color ranges for objects overlapped, we first used the exclusive color range to identify an object, and only used the wider range if a threshold number of object pixels was detected. Contextual color change was when the color space of an object was modified by other objects. For example, clouds modified the appearance of water because water reflects clouds. To account for this, we used if-else statements to modify how water pixels were identified when clouds were present. When objects changed temporally, we also used if-else statements to change object color space over time. For example, when the original, relatively broad range for water detection performed poorly during spring and fall we used if-else logic to narrow the color space, accounting for seasonal changes in water reflecting green, greening or brown vegetation. Rather than hard-code dates for switching object color assignment, we used a color index that tracked temporal change while still allowing for year to year variation, the Green Chromatic Coordinate (GCC). GCC is calculated as $\mathrm{G}_{\mathrm{a}} /\left(\mathrm{R}_{\mathrm{a}}+\mathrm{G}_{\mathrm{a}}+\mathrm{B}_{\mathrm{a}}\right)$ (Richardson et al., 2007), where $\mathrm{R}_{\mathrm{a}}, \mathrm{G}_{\mathrm{a}}$ and $\mathrm{B}_{\mathrm{a}}$ represent the average value of R, G, and B within the marsh ROI. GCC, a color-based phenology index, tracks the seasonal progress of vegetation green-up.

As the final step in the tuning process (Fig. 2, Step 4d), we compared early stage smart-classifier output with results from methods previously used on PhenoCam imagery, all in comparison to hand-estimated cloud cover quartiles from the training data. We evaluated the 90th percentile method and two solar illumination indices (BCC and WFI) (Ide and Oguma, 2010; Julitta et al., 2014; Sonnentag et al., 2012) to see if these indices, which are based on average ROI values, could improve classification accuracy. The 90th percentile method identifies optimal data as the maximum value of phenology indices, such as GCC, from within a 3-day moving window. Maximum GCC values are substituted for other values within the window, presumably selecting optimal data (the brightest scenes) to replace sub-optimal data. $\mathrm{BCC}$ is calculated as $\mathrm{B}_{\mathrm{a}} /\left(\mathrm{R}_{\mathrm{a}}+\mathrm{G}_{\mathrm{a}}+\mathrm{B}_{\mathrm{a}}\right)$ using average values within the marsh ROI. WFI uses both BCC and RCC 
$\left(\mathrm{R}_{\mathrm{a}} /\left(\mathrm{R}_{\mathrm{a}}+\mathrm{G}_{\mathrm{a}}+\mathrm{B}_{\mathrm{a}}\right)\right.$ and is calculated as $(\mathrm{BCC}-\mathrm{RCC}) /(\mathrm{BCC}+\mathrm{RCC})$, using average values within the sky ROI. Of these indices, only WFI improved the smart classifier. WFI increased smart classifier specificity for the cloud cover estimation by detecting scenes where smoke/grey haze on the horizon was misidentified as clouds. The final smart classifier therefore incorporates WFI as a scene context filter for the sky ROI, thereby removing false positives. Incorporating WFI into the smart classifier increased training specificity from $20 \%$ to $60 \%$ while retaining other smart classifier accuracy measures.

We consider this a "smart" classifier because we ultimately created rules that changed the classification scheme based on within scene content, such as reflections from nearby objects, temporal change and object color overlap.

The finalized smart classifier automatically classifies the images without user input other than a date range. It estimates the percent cover of all tracked objects as continuous variables, and outputs a file summarizing the classification results. We compared the results of the smart classifier to two independent sets of hand-classified scenes: testing data $(\mathrm{N}=124)$ and validation data $(\mathrm{N}=65)$ (Fig. 2: Step 5). Although neither were used for model training, the validation data were created after the smart classifier was finalized to increase cross-validation.

To present the smart classifier, we calculated GCC for all scenes and compared results for subsets of images identified as optimal, flooded, and cloudy. An important feature of the smart classifier is that end-users can choose any cut-off level to filter data. Since our application was vegetation analysis, we chose to define "optimal" scenes as those with $>99 \%$ vegetation in the marsh ROI and <20\% clouds in the sky ROI. We defined scenes with < $1 \%$ water in the marsh ROI as "flooded" and those with $>70 \%$ clouds in the sky ROI as "cloudy".

Finally, we compared GCC phenology time-series for smart classifier-identified optimal PhenoCam scenes with those from BCC, WFI, and the 90the percentile approaches, all of which are existing methods for filtering PhenoCam imagery. Although WFI was incorporated into the smart classifier, it is also used as a stand-alone method and was therefore included in this comparison. To build a BCC index, we started with Julitta et al.'s (2014) equation, which accepted scenes for downstream analysis if the BCC values were within the range of the daily mean $+/-$ a fraction of the seasonal standard deviation. We optimized Julietta et al.'s (2014) index for our site by modifying the window size used to calculate the local mean and standard deviation of BCC in the marsh ROI and iteratively compared different options to the training data. The optimized BCC index selected scenes with marsh ROI BCC values $\left(\mathrm{BCC}_{\mathrm{i}}\right)$, such that: $\mathrm{BCC}_{\mathrm{lm}}-0.04 * \mathrm{BCC}_{\mathrm{lsd}}<\mathrm{BCC}_{\mathrm{i}}<\mathrm{BCC}_{\mathrm{lm}}+0.04 * \mathrm{BCC}_{\mathrm{lsd}}$, where $\mathrm{BCClm}_{1 m}$ and $\mathrm{BCC}_{1 s d}$ are the local mean and local 
standard deviation of BCC within a 30-d moving average window. The optimized WFI index was calculated for the sky ROI (Ide and Oguma, 2010). To optimize WFI, all possible WFI values were compared with hand-estimated cloud cover and index cut-offs that maximized accuracy of cloud cover estimation were selected. Finally, we applied the 90th percentile method (Sonnentag et al., 2012), which selects maximal GCC within a 3-d moving average window as optimal data and substitutes it for other values within the window. We used smart classified cloud cover to compare clouds from data corresponding to the 90th percentile of GCC with those from the 10th percentile. If the 90th percentile method selects days with less cloud cover than the full data set, it should have lower mean cloud cover than the 10 th percentile.

\section{Results}

Object color properties could be exploited for pixel classification, but water and clouds overlapped with other objects in their widest possible color ranges (Supplementary Information: Table SI1), resulting in low accuracies for unsupervised classification (Table 1).

When we applied the smart classifier, detection accuracy was $>92 \%$ for all objects within training images, and $>86 \%$ within testing and validation images. Prediction accuracy of scenes with optimal vegetation cover was $96 \%$ for validation data. The smart classifier was more accurate for detecting and quantifying flooding than measured tide height in an adjacent creek, which explained only $20 \%$ of flooding variation because it did not account for wind or local topography (data not shown).

The smart classifier identified 987 scenes as "optimal" for vegetation analysis, 4215 as "cloudy", and 2117 as "flooded" based on our definitions. GCC values for optimal images had lower variability than the full dataset (Fig. 3). Cloudy scenes had slightly greater GCC values than optimal images (mean $\pm \mathrm{SD}=0.37 \pm 0.02$ and $0.35 \pm 0.02$, respectively), whereas flooded images generally added random noise to GCC values

Other approaches for filtering PhenoCam imagery had varied success. The BCC index reduced data variation and selected data corresponding to the local mean tendency (Fig. 4). However, it still did not accurately reflect handestimated cloud cover within the training set (detection accuracy 0.63 , sensitivity 0.98 , specificity 0.03 ). It also selected the fewest data points for downstream analysis (Fig. 5). WFI out-performed BCC (Fig. 4, 5). Applying the WFI cut-offs in Table 2 allowed us to estimate training data cloud cover with the following accuracies: detection, 92; sensitivity, 92; specificity, 80; quantity, 71. However, the smart classifier's pixel-based estimate of cloud quartiles was 
more accurate than WFI's sky ROI approach for all measures except specificity (Table 1). The 90th percentile method was the least effective previous approach. The 3-day 90 th percentile of GCC had mean cloud cover of $69 \pm 41 \%$ as compared to the 3 -day 10 th percentile $(57 \pm 42 \%)$ and optimal imagery $(4 \pm 5 \%)$. Therefore, the maximal GCC approach often selected data from cloudy scenes and substituted these for optimal data (Fig. 3). The 90th percentile phenology time-series also had the greatest noise compared with other methods (Fig. 5).

\section{Discussion}

The smart classifier uses within-scene information from image time-series to automatically and effectively filter data for image analysis. It relies on rules that provide different RGB ranges for object classes and account for RGB differences resulting from scene context. In our application, phenology analysis of the "GCESapelo" PhenoCam on Sapelo Island, GA, USA, applying the smart classifier resulted in higher object classification accuracy than unsupervised approaches. Results also compared well with hand-classified images (detection accuracy $>86 \%$ and quantity accuracy $>78 \%$ ). Therefore, we suggest a pixel classification approach is useful, especially for images exhibiting high seasonal or within-scene heterogeneity. To our knowledge, this is a unique approach for automated digital analysis of PhenoCam data.

The smart classifier worked well at this site, effectively filtering out scenes with poor solar illumination (cloudy) or non-target objects (flooded), thereby resulting in a subset of optimal scenes with reduced GCC variation. Previous approaches for filtering PhenoCam data were less accurate for this site. The BCC (Julitta et al., 2014) filtered data that departed sharply from local BCC means, and these often were from flooded or cloudy scenes. However, many cloudy and flooded images had the same BCC range as optimal data, so the filtered data set was neither specific nor accurate for indexing solar illumination. BCC also imperfectly indexes marsh flooding because the color space of water is changeable. Although WFI does not address flooding, it was effective at separating cloudy images and we would recommend this index at sites where a smart classifier is not available. However, we obtained the greatest accuracy for identifying cloudy scenes when we used a smart classifier that incorporated WFI. The 90th percentile approach substituted cloudy scenes with maximal GCC for optimal data. It should therefore be used with caution as it may document weather as opposed to vegetation trends.

To adapt a smart classifier to a new site, one might follow the steps outlined in Fig. 2. If the new classifier will track vegetation, one could start with the Appendix 1 code, adding code for tracking new objects and removing code for 
those not found at the new location. Color properties of vegetation probably will be consistent across biomes because vegetation color is determined by plant pigments common across vegetation types. For new classifiers, classification accuracy across training data can identify where adjustments should be made and final accuracy can be verified with testing and validation data.

The smart classifier produces time-series for all classified objects, such as clouds and flooding, which could be useful for tracking local weather conditions or for lending insight into interpreting vegetation patterns. It could also be adapted for other applications such as monitoring small-scale flood patterns, tracking new objects, such as animals, or selecting high quality satellite data (cloud and flood free) for coastal wetland analysis.

Acknowledgements. The Georgia Coastal Ecosystems LTER is supported by the National Science Foundation (OCE12-37140). We thank Wade Sheldon, Jacob Shalack and the National PhenoCam Network for managing and curating the GCE PhenoCam. This is contribution 1046 of the University of Georgia Marine Institute.

Software and data availability . Code for the smart classifier is included as Supplementary Information; we can be contacted about the program via author contract information. The smart classifier was developed in $\mathrm{R}$ and is freely available for others to use, build on, or improve. Model training imagery are at:

phenocam.sr.unh.edu/webcam/sites/gcesapelo/

\section{References}

Bennett, N.D., et al., 2013. Characterising performance of environmental models. Environmental Modelling \& Software 40, 1-20. doi:10.1016/j.envsoft.2012.09.011

Bordese, M., Alini, W., 2013. biOps: Image processing and analysis. R package version 0.2.2. https://CRAN.Rproject.org/package=biOps

Ide, R., Oguma, H., 2010. Use of digital cameras for phenological observations. Ecological Informatics, Special issue on advances of ecological remote sensing under global change 5, 339-347. doi:10.1016/j.ecoinf.2010.07.002

Julitta, T., et al., 2014. Using digital camera images to analyse snowmelt and phenology of a subalpine grassland. Agricultural and Forest Meteorology 198-199, 116-125. doi:10.1016/j.agrformet.2014.08.007

Kaufman, L., Rousseeuw, P.J., 2009. Clustering Large Applications (Program CLARA), in: Finding Groups in Data: An Introduction to Cluster Analysis. John Wiley \& Sons, Hoboken, NJ, USA, pp. 126-163.

Kuhn, M., 2008. Building predictive models in R using the caret package. Journal of Statistical Software 28. 
Richardson, et al., 2007. Use of digital webcam images to track spring green-up in a deciduous broadleaf forest.

Oecologia 152, 323-334. doi:10.1007/s00442-006-0657-z

Romero-Ramirez, A., Grémare, A., Desmalades, M., Duchêne, J.C., 2013. Semi-automatic analysis and interpretation of sediment profile images. Environmental Modelling \& Software 47, 42-54. doi:10.1016/j.envsoft.2013.04.008

Sonnentag, O., et al., 2012. Digital repeat photography for phenological research in forest ecosystems. Agricultural and Forest Meteorology 152, 159-177. doi:10.1016/j.agrformet.2011.09.009

Zhou, Q., Robson, M., 2001. Automated rangeland vegetation cover and density estimation using ground digital images and a spectral-contextual classifier. Internat. J. Remote Sensing 22, 3457-3470. 10.1080/01431160010004504 


\section{TABLES}

Table 1: Classification accuracy of an unsupervised classifier (applied to validation images, $N=$ 65 ) vs the smart classifier (applied to training, $N=201$; testing $N=124$; and validation images $N=$ $65)$ for quantifying objects within digital image time series of tidal salt marshes.

\begin{tabular}{|c|c|c|c|c|}
\hline accuracy & unsupervised & training & testing & validation \\
\hline \multicolumn{5}{|l|}{ Vegetation } \\
\hline detection & 100 & 100 & 100 & 100 \\
\hline sensitivity & 100 & 100 & 100 & 100 \\
\hline specificity & - & - & - & - \\
\hline quantity & 66 & 91 & 92 & 83 \\
\hline optimal cover & 88 & 95 & 90 & 96 \\
\hline \multicolumn{5}{|l|}{ Water } \\
\hline detection & 78 & 92 & 88 & 86 \\
\hline sensitivity & 89 & 85 & 73 & 85 \\
\hline specificity & 73 & 95 & 94 & 87 \\
\hline quantity & 62 & 91 & 92 & 82 \\
\hline \multicolumn{5}{|l|}{ Shadows } \\
\hline detection & 82 & 93 & 90 & 91 \\
\hline sensitivity & 54 & 88 & 85 & 93 \\
\hline specificity & 92 & 95 & 91 & 90 \\
\hline quantity & 78 & 100 & 100 & 100 \\
\hline \multicolumn{5}{|l|}{ Fog } \\
\hline detection & 88 & 94 & 94 & 92 \\
\hline sensitivity & 40 & 83 & 58 & 64 \\
\hline specificity & 100 & 95 & 98 & 98 \\
\hline quantity & 78 & 100 & 100 & 100 \\
\hline \multicolumn{5}{|l|}{ Clouds } \\
\hline detection & 78 & 96 & 88 & 88 \\
\hline sensitivity & 76 & 96 & 91 & 90 \\
\hline specificity & 100 & 60 & 43 & 67 \\
\hline quantity & 62 & 83 & 79 & 78 \\
\hline
\end{tabular}


Table 2: Optimized WFI values for predicting hand-estimated cloud cover quartiles.

\begin{tabular}{ll}
\hline quartile & WFI \\
\hline $0 \%$ & $>0.12$ \\
$>0-25 \%$ & $0.120-0.080$ \\
$25-50 \%$ & $0.080-0.042$ \\
$50-75 \%$ & $0.042-0.025$ \\
$75-99 \%$ & $0.025-0.009$ \\
$>99 \%$ & $<0.009$ \\
\hline
\end{tabular}




\section{FIGURES}

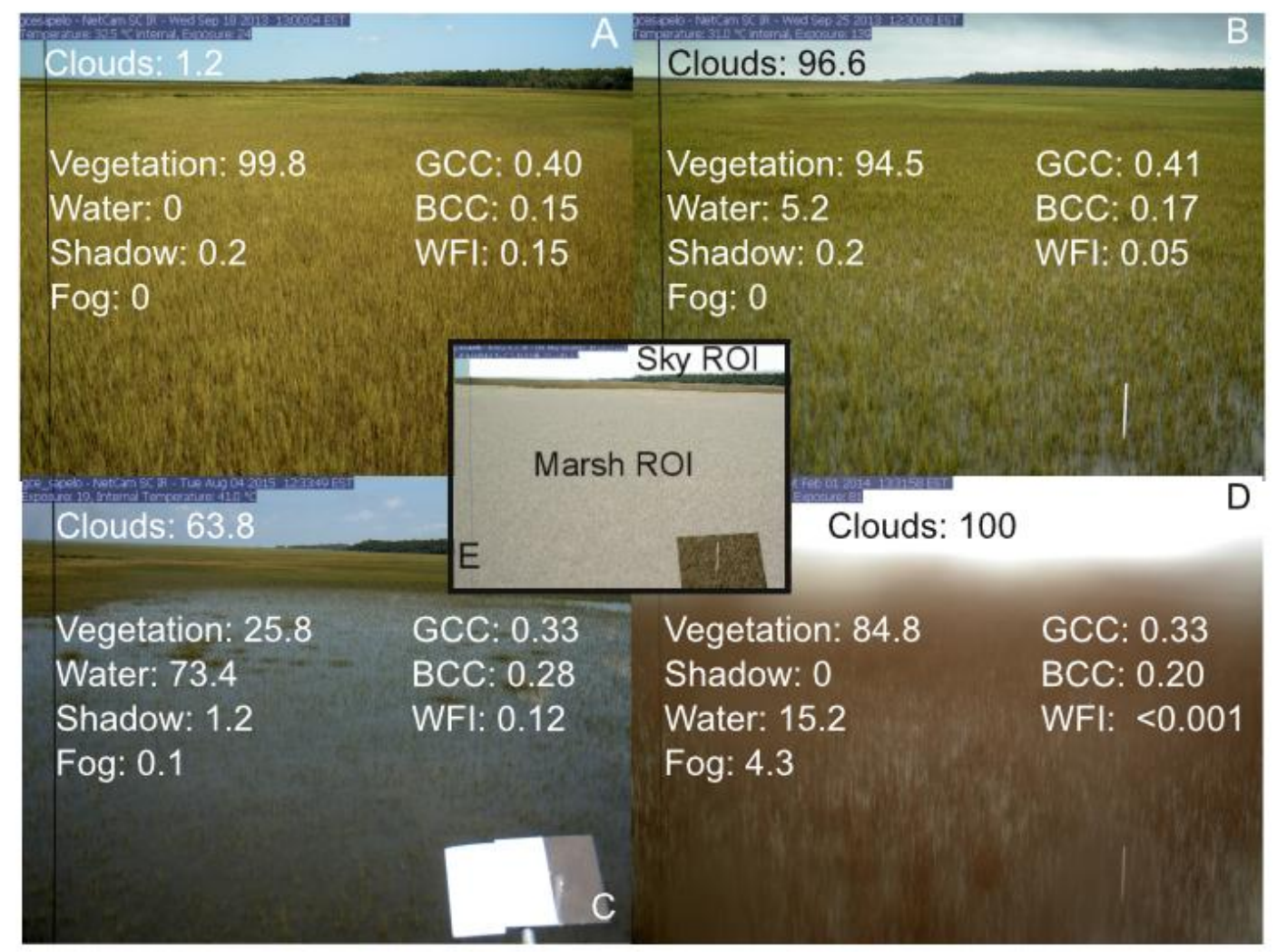

Fig. 1: Images from the GCESapelo PhenoCam, showing scenes with optimal data for vegetation analysis (A), with low flooding (B), high flooding (C), and fog and clouds (D). Smart classifier estimated percent cover is provided, as well as phenology (GCC) and weather indices (BCC and WFI) discussed within the text. The regions of interest (ROIs) are shown in the inset $(\mathrm{E})$. 


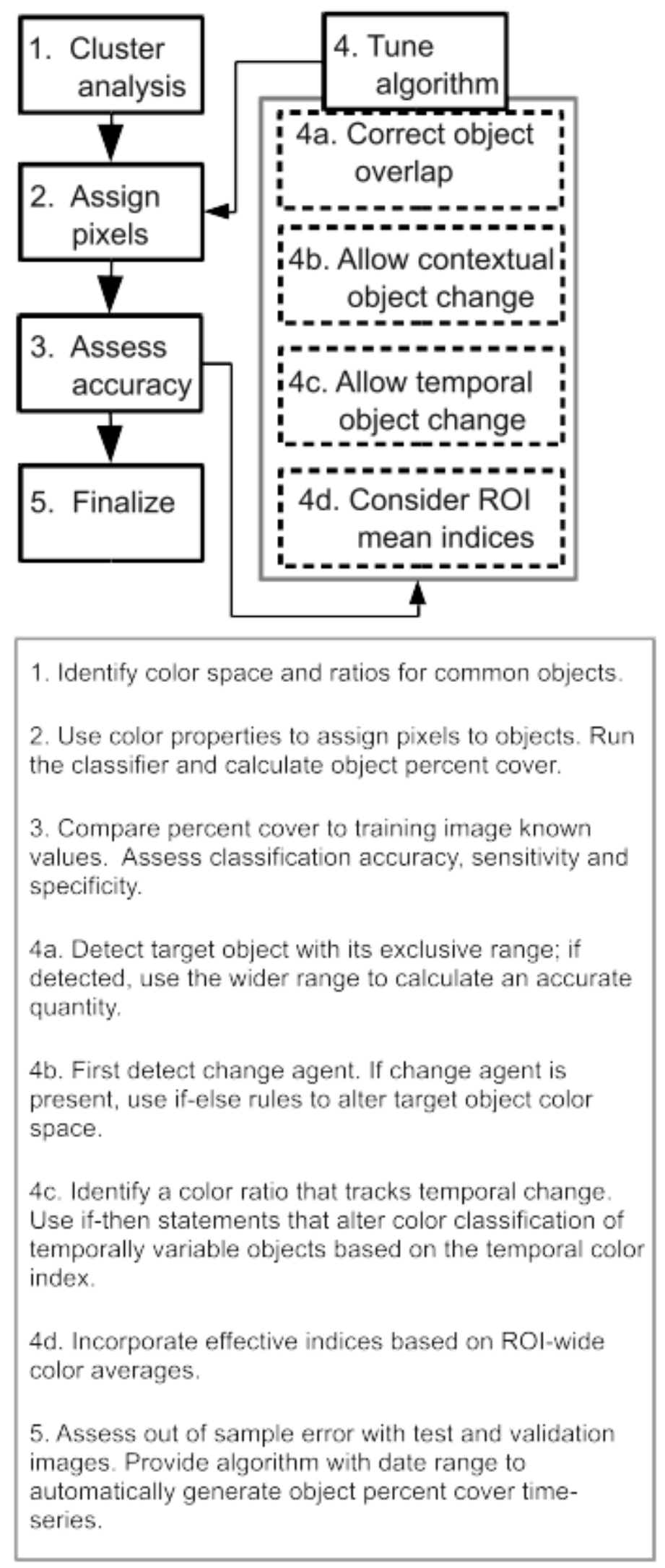

Fig. 2: The conceptual steps used to create and validate the smart classifier. 


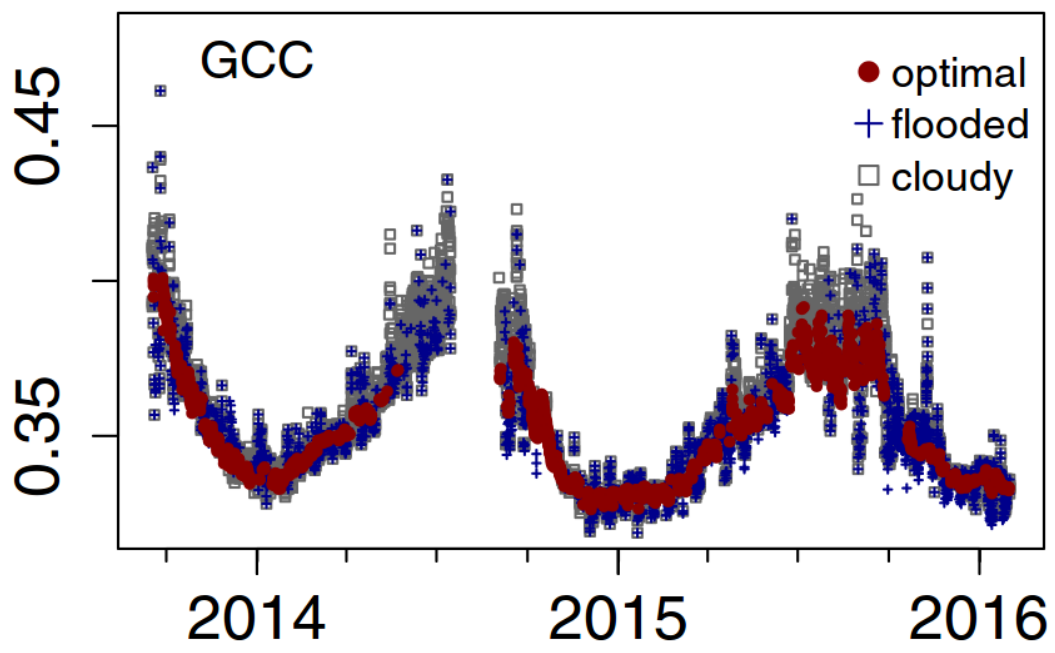

Fig. 3: The phenology index, GCC tracked through time for the GCESapelo PhenoCam. Optimal scenes had > 99\% vegetation and $<20 \%$ clouds; cloudy scenes had $>\mathbf{7 0} \%$ clouds; flooded scenes had $>\mathbf{5 \%}$ water. Analyses were run on the full dataset, but to avoid clutter only points that met the above criteria were shown. 

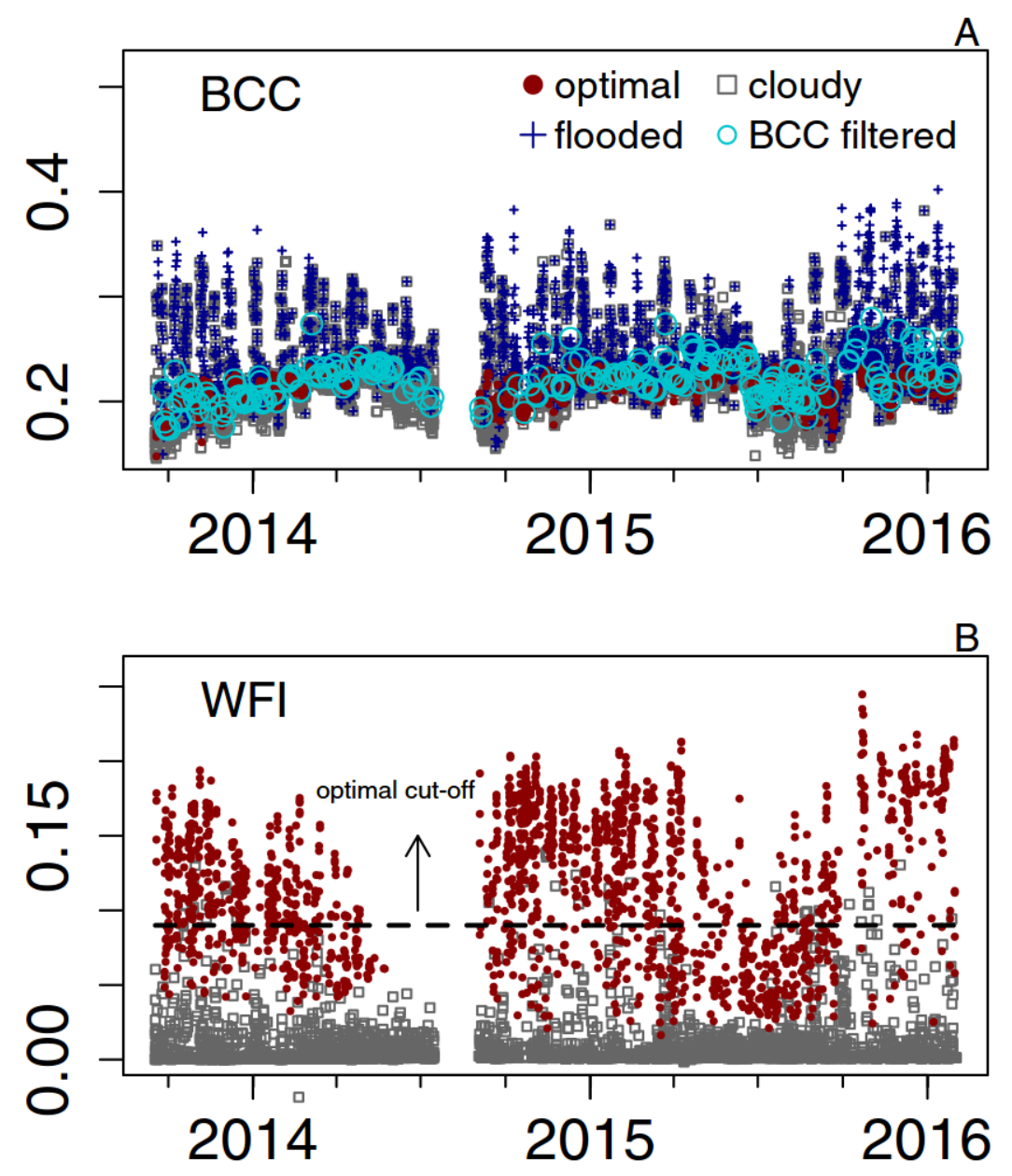

Fig. 4: Solar illumination indices, BCC (A) and WFI (B). BCC is a marsh ROI index and BCC filtered pixels were those close to the local BCC mean (see methods). BCC filtering is compared with smart classified cloudy, flooded and optimal data. WFI is a sky ROI index and is compared with smart-identified cloudy and optimal data. WFI filtered data are those above an optimized WFI cut-off (shown as dashed line). 

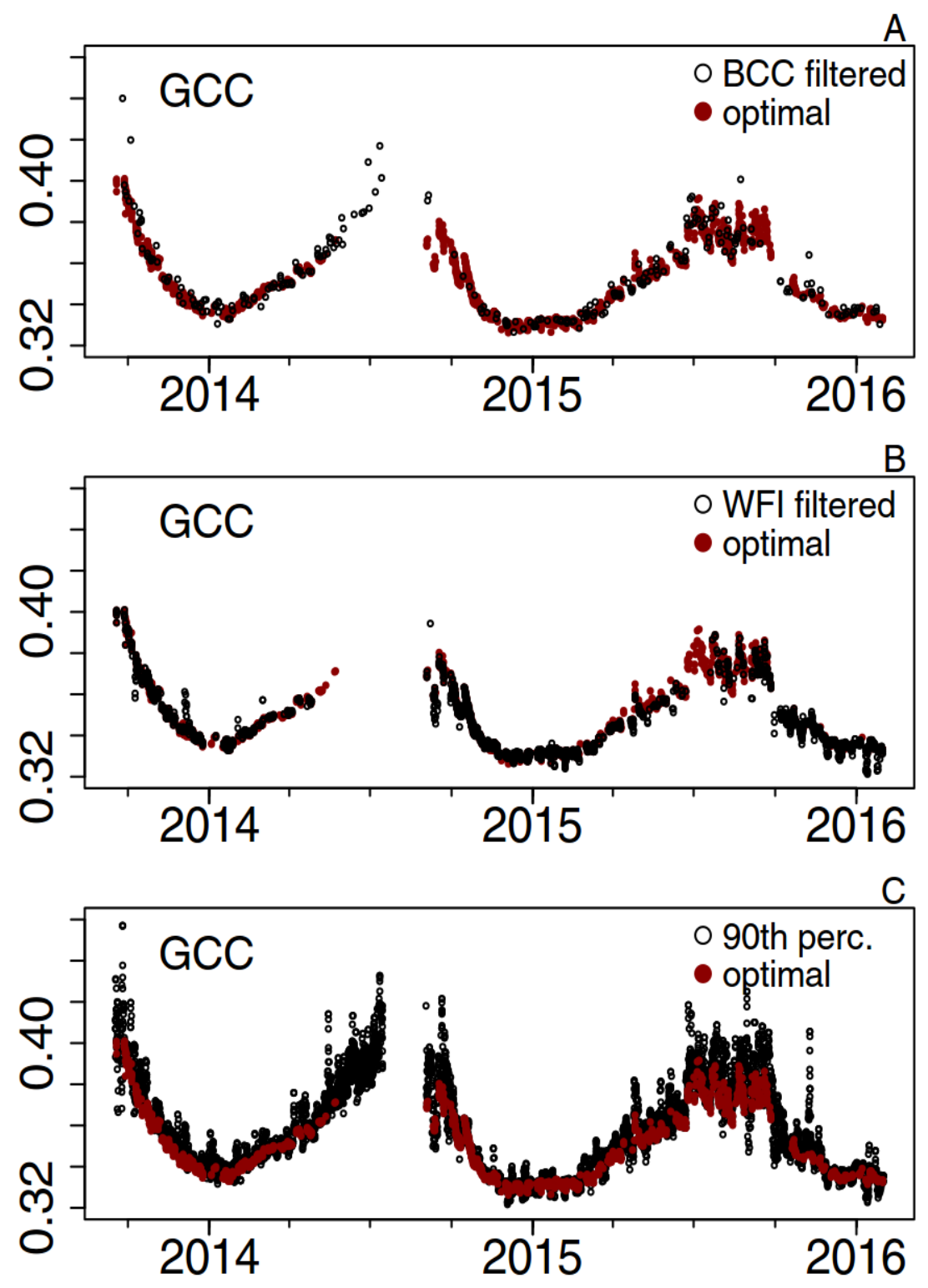

Fig. 5: GCC time-series estimated via BCC filtering (A), WFI filtering (B), or the 90th percentile method (C). Each is compared with GCC extracted directly from smart-identified optimal images. 